\title{
Use Case
}

National Cancer Institute

\section{Source}

National Cancer Institute. Use Case. NCI Thesaurus. Code C81930.

A document that describes the interaction between a user (or other initiator of the interaction) and a system, represented as a sequence of simple steps leading to a particular goal. 